\title{
As Representações Sociais de pessoa velha construídas por adolescentes
}

\section{Social Pepresentations of eddely built by teenagers}

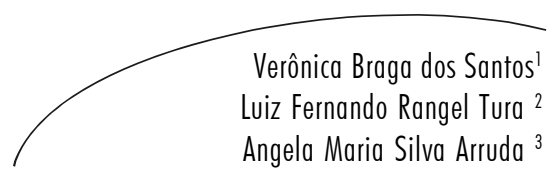

Resumo

O envelhecimento populacional é fenômeno relevante no mundo e desencadeia repercussões na dinâmica social, viabilizando a existência de representações sociais que deem sentido e orientem os grupos sociais acerca do envelhecimento humano. Com base na abordagem estrutural, foram investigadas a estrutura e a organização dos conteúdos da representação social sobre Pessoa Velha construída por adolescentes. Realizou-se um teste de evocação livre de palavras com a expressão "pessoa velha" e aplicou-se um questionário com perguntas abertas acerca de crenças, atitudes, normas, valores e práticas relacionadas ao processo de envelhecimento e ao idoso e a caracterização sócio-demográfica. Participaram 137 estudantes do $2^{\circ}$ ano do Ensino Médio de uma instituição federal de ensino no Rio de Janeiro; idade entre 15-19 anos (média 16,19 anos); e maioria do sexo masculino (65,44\%). Os elementos com atributos de centralidade foram: Avô, Idoso e Experiência. O sistema periférico era composto por: Sabedoria, Cabelo Branco, Respeito, Rugas, Aposentado, Doença, Personagens, Conbecimento, Bengala, Antigo, Remédio, Dificuldades, Morte, Acabado, Legal, Óculos, Pai-Mãe-Tia, Tempo e o sistema intermediário por: Idade, Chata, Gratuidades e Senhora. Os principais organizadores da representação foram: Avô e Idoso. Destacam-se dois sentidos atribuídos à pessoa velha: os elementos relacionados a $A v \hat{o}$ oferecem concretude à imagem de pessoa velha. E a Idoso agregam-se sentidos relativos a uma construção normativa da categoria Idoso, ensejando respeito e valorização. O estudo pode servir de subsídio para a elaboração de políticas de saúde, auxiliando na orientação de medidas direcionadas aos idosos e aos aspectos do envelhecimento humano.

\footnotetext{
Instituto de Estudos em Saúde Coletiva. Universidade Federal do Rio de Janeiro. Rio de Janeiro, RJ, Brasil.

2 Faculdade de Medicina. Universidade Federal do Rio de Janeiro. Rio de Janeiro, RJ, Brasil.

3 Instituto de Psicologia. Universidade Federal do Rio de Janeiro. Rio de Janeiro, RJ, Brasil

Palavras-chave: Pessoa Velha. Representações Sociais. Envelhecimento. Adolescentes. Saúde do idoso. Qualidade de vida 


\section{Abstract}

Population aging is an important phenomenon in the world, with impact on the social dynamics, allowing for the existence of social representations which give sense and guide social groups concerning human aging. This study focused on the social representations of Old person built by teenagers, analyzing their structure and organization. Based on a structural approach, a test was conducted to freely evoke words related to the expression "old person" and a questionnaire was given to elicit answers regarding beliefs, attitudes, rules, values and practices related to the aging process and to the elderly and their socio-demographic characterization. Took part in the study 137 high school sophomores from a federal institution in Rio de Janeiro, ages 15 to 19 (average 16.19 years old), mostly male (65.44\%). The elements which stood out as central were: Grandparent, Elderly and Experience. The peripheral system was composed of: Wisdom, Grey Hair, Respect, Wrinkles, Retired, Disease, Characters, Knowledge, Cane, Ancient, Medicine, Difficulties, Death, Worn-out, Nice, Glasses, Father-Mother-Aunt, Time and the intermediary system was composed of: Age, Annoying, Gratuities and Madam. The main representation organizers were Grandparent and Elderly, which suggest the existence of two focuses of meanings given to the old person. The elements related to Grandparent offer concreteness to the image of old person. And those associated to Elderly add meanings related to a normative construction of the Elderly category, which brings about respect and value, in opposition to a negative or belittling connotation associated to the term "old". The study may provide support for the development of health policies, serving as guide for measures taken in favor of the elderly and aspects of human aging.
Key words: Old Person. Social Representations. Aging. Teenagers. Health of the Elderly. Quality of Life.

\section{INTRODUÇ̃̃OO}

$\mathrm{Na}$ contemporaneidade, era de avanços nas tecnologias de comunicação de massa, há maior velocidade de divulgação e de acesso às informações e acontecimentos do dia a dia. Uma gama variada de temas causa estranhamento e pode se tornar presente nas conversas e nas interações. ${ }^{1}$ Forma, assim, um substrato diversificado da experiência vivida que pode ser utilizado para a manutenção, reformulação e formação de representações sociais.

O uso da Teoria das Representações Sociais (TRS) visa a compreender as interpretações e os sentidos que os grupos e sujeitos têm sobre objetos sociais relevantes, com o enfoque no saber construído no cotidiano dos grupos sociais - o conhecimento do senso comum. ${ }^{2}$ Nesse âmbito surgem aspectos do envelhecimento populacional, processo que se torna cada vez mais relevante no mundo e na sociedade brasileira, que despertam interesse por suas implicações nos diversos setores da sociedade, como na área da saúde e, consequentemente, por suas repercussões na dinâmica social e no cotidiano. ${ }^{3-7}$

Assim, o tema do envelhecimento propicia condições para a existência de representações sociais que possam dar sentido, orientar e conduzir os grupos sociais. As representações formam um saber prático tanto por estarem inseridas na experiência, que envolve um contexto histórico, cultural e espacial, quanto por orientarem as comunicações e condutas dos sujeitos. ${ }^{1}$ Por sua natureza e por estarem inseridas em uma sociedade em que o aumento da expectativa de vida propiciará crescente convívio familiar entre mais de duas ou três gerações, ${ }^{5}$ as representações sociais construídas por adolescentes sobre os aspectos do envelhecimento, da velhice e do ser idoso tornam-se importantes focos de estudo, uma vez que esses sujeitos terão que lidar com o próprio envelhecimento e o de outras pessoas. 
A literatura sobre os sentidos construídos acerca do envelhecimento, idoso, velho e velhice, assinala diferenças e aproximações de significados entre grupos etários distintos. Em estudos sobre concepções, imagens e representações construídas por jovens, destacam-se conteúdos relativos a perdas nas condições e atividades corporais, como surgimento de doenças, desgaste, enfraquecimento, incapacidades, dependência, fim, decadência, inatividade sexual, fragilidade e vulnerabilidade. E ainda, aspectos psicológicos que remetem à solidão, sofrimento, desânimo, frustração e a condições do idoso ou velho na relação e inserção nos grupos sociais, como discriminação social, abandono, perda de credibilidade, inadaptabilidade, desvalorização social, marginalização e passividade..$^{8-13}$

No entanto, na maioria desses estudos, os conteúdos de perdas, desgastes e de desvalorização do idoso são contrapostos à sabedoria, experiência e ao conhecimento adquiridos com o envelhecer. Estabelece-se, assim, uma relação em que sentidos de perdas e de desvalorização são amenizados e compensados por ganhos. ${ }^{8-14}$

A partir da perspectiva da TRS, o presente trabalho tem como objetivo investigar a existência e as características de representações sociais sobre Pessoa Velha construídas por adolescentes.

\section{PROCEDIMENTOS METODOLÓGICOS}

A investigação seguiu os procedimentos metodológicos relativos à abordagem estrutural das representações sociais, que enfatiza os conteúdos cognitivos das representações, com o objetivo de identificar sua estrutura e organização. As representações sociais são consideradas um sistema sócio-cognitivo que pode ser ao mesmo tempo rígido e flexível, estável e móvel. Para contemplar estas características aparentemente contraditórias, a abordagem estrutural propõe que uma representação social é formada por dois sistemas distintos, mas complementares - o central e o periférico. ${ }^{15}$
A ideia essencial é que os elementos são hierarquizados e toda representação está organizada em torno de um núcleo central, que determina sua significação e organização interna. ${ }^{15}$ Abric $^{16}$ explicita as duas funções do sistema central: a geradora, por ser o seu conteúdo o elemento pelo qual se cria ou transforma o significado dos outros elementos; e a organizadora, por unificar e estabilizar a representação, de forma que a mudança de seu núcleo central desencadeia a transformação da representação. $O$ sistema central caracteriza-se, entre outros aspectos, por seus elementos serem estáveis e seu conteúdo estar ligado à memória coletiva e à história do grupo.

Além dessas funções, há duas dimensões relacionadas ao sistema central: a dimensão funcional, em que seus elementos estarão voltados para a realização de tarefas; e a dimensão normativa, em situações que envolvem dimensões sócio-afetivas, sociais ou ideológicas. ${ }^{15} \mathrm{O}$ sistema periférico está na interface entre realidade concreta e sistema central; sua dimensão é funcional, por possibilitar a ancoragem da representação na realidade do momento. Isso é permitido por sua flexibilidade, que regula e adapta o sistema central a uma situação concreta. Assim, o sistema periférico é mais determinado por características do contexto imediato. ${ }^{15}$

Desse modo, pretende-se investigar as representações sociais com base na abordagem estrutural de forma a identificar os conteúdos e a organização de sua estrutura. $\mathrm{O}$ instrumento de coleta de dados foi formado por um teste de evocação livre de palavras (TEP) de uso frequente nesse tipo de abordagem ${ }^{17-21}$ e de um questionário com perguntas abertas que permite a caracterização sócio-demográfica e a exploração de crenças, atitudes, normas, valores e práticas acerca do processo de envelhecimento e do idoso.

Neste estudo, a frase indutora do TEP foi "quais as quatro primeiras palavras que vêm a sua cabeça quando ouve falar em 'pessoa velha'”. Em seguida, foi solicitado aos sujeitos que assinalassem as duas palavras que considerassem como mais importantes e que justificassem as respectivas 
escolhas. A análise dos dados seguiu a proposta de Vergès, ${ }^{22}$ que leva em consideração as dimensões individual (ordem de evocação) e coletiva (frequência) envolvidas no processo de evocação, possibilitando identificar, assim, os conteúdos da estrutura da representação em estudo.

Além disso, utilizou-se a análise de similitude para o estudo da organização dos elementos que compõem a representação investigada. ${ }^{23} \mathrm{~A}$ análise das respostas às perguntas abertas foi baseada na análise categorial temática como descrita por Bardin. $^{24}$

O universo estudado foi constituído por estudantes matriculados em 2009 no $2^{\circ}$ ano do Ensino Médio de uma das unidades de uma instituição federal de ensino no Rio de Janeiro. A aplicação dos questionários ocorreu nas salas de aula, em todas as turmas dos turnos matinais e vespertinos.

Durante a investigação, procurou-se contemplar as exigências sobre as pesquisas que envolvam seres humanos, com cuidados metodológicos relativos aos princípios éticos, considerando a relevância social, viabilidade, autonomia do sujeito, preservação da confidencialidade dos dados e resultados e avaliando sua beneficência. ${ }^{25} \mathrm{O}$ projeto foi analisado e aprovado pelo Comitê de Ética em Pesquisa do Instituto de Estudos em Saúde Coletiva e um termo de consentimento livre e esclarecido foi apresentado aos participantes.

\section{RESULTADOS E DISCUSSÃO}

O grupo estudado foi constituído por 137 adolescentes, com amplitude de idade entre $15 \mathrm{e}$
19 anos, média igual a 16,19 e mediana de 16 anos. A maioria é do sexo masculino $(65,44 \%)$.

A partir de uma "leitura flutuante" do corpus obtido, foram unificadas as formas singular/ plural, masculino/feminino, e agregados os sinônimos, com base nas evocações de maior frequência, de forma a homogeneizar o material. ${ }^{23}$ O TEP foi respondido pelos 137 participantes, totalizando 548 evocações. Seguiu-se com a verificação das frequências $(F)$ dos elementos evocados em cada posição e a média de frequência $(\mathrm{Fm})$. Constatou-se que os dez mais frequentes Avô (83), Idoso (63), Experiência (40), Sabedoria (22), Cabelo Branco (17), Respeito (17), Idade (16), Rugas (16), Aposentado (14) e Doença (14), - correspondiam a $55,1 \%$ do total do material evocado.

O próximo passo consistiu no cálculo da ordem média de evocação (ome) dos diversos elementos e as médias das ordens médias de evocação (OME). A análise combinada desses dois parâmetros (Fm e OME) possibilitou a distribuição dos diversos elementos em um gráfico de dispersão em que o cruzamento das linhas relativas a Fm e OME permite sua divisão em quatro quadrantes. Neste estudo, a Fm foi igual a 30 e a OME igual a 2,5. Assim sendo, os elementos com frequência igual ou maior a 30 e OME menor do que 2,5 encontram-se no quadrante superior esquerdo, constituindo o sistema central da representação. Já o quadrante inferior direito é composto pelos elementos do sistema periférico, aqueles de menor frequência e maior ordem de evocação, isto é, mais tardiamente evocado. Os quadrantes restantes, superior direito e inferior esquerdo, contêm os elementos do sistema intermediário ou da periferia próxima ao sistema central ${ }^{26}$ (quadro 1). 
Quadro 1 - Distribuição dos elementos segundo frequência de evocação e ordem média de evocação realizadas pelos adolescentes. Rio de Janeiro, RJ. 2009.

\begin{tabular}{|c|c|c|c|c|c|c|}
\hline Fm & Elementos & $\mathrm{f}$ & Ome $<2,5$ & Elementos & $\mathrm{f}$ & Ome $>=2,5$ \\
\hline \multirow{3}{*}{$>=30$} & AVO & 83 & 2,217 & & & \\
\hline & IDOSO & 63 & 1,683 & & & \\
\hline & EXPERIÊNCIA & 40 & 2,225 & & & \\
\hline \multirow{18}{*}{$<30$} & IDADE & 16 & 1,750 & SABEDORIA & 22 & 2,864 \\
\hline & CHATA & 12 & 1,917 & CAB-BRANCO & 17 & 3,000 \\
\hline & GRATUIDADES & 5 & 2,400 & RESPEITO & 17 & 2,941 \\
\hline & SENHORA & 5 & 2,400 & RUGAS & 16 & 2,688 \\
\hline & & & & APOSENTADO & 14 & 2,786 \\
\hline & & & & DOENCA & 14 & 3,000 \\
\hline & & & & PERSONAGENS & 12 & 3,000 \\
\hline & & & & CONHECIMENTO & 11 & 2,727 \\
\hline & & & & BENGALA & 10 & 2,700 \\
\hline & & & & ANTIGO & 8 & 2,750 \\
\hline & & & & REMEDIO & 8 & 3,125 \\
\hline & & & & DIFICULDADES & 7 & 2,571 \\
\hline & & & & MORTE & 7 & 3,429 \\
\hline & & & & ACABADO & 6 & 3,000 \\
\hline & & & & LEGAL & 6 & 2,667 \\
\hline & & & & OCULOS & 6 & 3,333 \\
\hline & & & & PAI-MAE-TIA & 5 & 2,600 \\
\hline & & & & TEMPO & 5 & 3,200 \\
\hline
\end{tabular}

Os elementos que apresentaram atributos de centralidade são $A v \hat{o}$, Idoso e Experiência, enquanto os que compõem o sistema periférico são Sabedoria, Cabelo Branco, Respeito, Rugas, Aposentado, Doença, Personagens, Conbecimento, Bengala, Antigo, Remédio, Dificuldades, Morte, Acabado, Legal, Óculos, Pai-Mãe-Tia, Tempo. O sistema intermediário foi composto pelos elementos Idade, Chata, Gratuidades e Senhora.

Ao examinar de forma mais atenta a composição do sistema periférico, foi possível formar subconjuntos que apontam aspectos por vezes distintos, mas também complementares, relacionados a uma pessoa velha. Os elementos de descrição física são os mais frequentes no sistema periférico. Incluem a aparência corporal - Cabelo Branco, Rugas, Acabado - querendo refletir as transformações físicas, a flacidez, a perda $\mathrm{da}$ beleza, além de acessórios - Bengala e Óculos - que podem estar relacionados à dificuldade de mobilidade, à dependência ou às dificuldades funcionais. A presença de Doença, Dificuldade, Remédio e Morte pode refletir condições patológicas frequentemente observadas na velhice, que impõem limitações e obrigam os idosos a tratamentos por vezes prolongados e que podem acarretar risco de vida.

Os elementos Morte, Tempo, Antigo e Aposentado também podem estar associados, de forma que a longa duração dos anos vividos ao mesmo tempo justificaria a proximidade da morte e caracterizaria a pessoa velha como antiga, que após cumprir suas obrigações de trabalho está aposentada.

A presença dos elementos Tempo, Antigo, Sabedoria, Conhecimento, Legal e Respeito no sistema periférico pode significar que, para pessoas mais vividas, a diversidade de situações permitiu a interação com diferentes formas de conhecimento. Essa condição pode orientar atitudes e práticas 
dos adolescentes, como as de manter uma relação de respeito e de admiração com a pessoa velha.

A associação de pessoa velha tanto com pessoas de convivência mais próxima dos sujeitos nas relações familiares quanto com aquelas que são conhecidas no próprio grupo social ou tem destaque nacional, por sua visibilidade ou importância na função que exercem ou exerceram (jogadores de futebol, artistas), é sugerida com a presença dos elementos Pai-Mãe-Tia e Personagens.

$\mathrm{Na}$ busca de outros indícios ${ }^{27}$ de centralidade, foi solicitada a indicação de duas palavras que julgassem mais importantes entre as evocações realizadas. Observou-se que a frequência dos elementos Avô, Experiência e Idoso foi igual a 49, 31 e 26, respectivamente. Comparando-se as duas frequências, de evocação e de importância, verificou-se que a diferença era menor do que $50 \%$ para Avô e Experiência. Assim, esses elementos agregam mais um indício de que sejam componentes do sistema central..$^{28}$

Ao verificar os elementos componentes da estrutura da representação social construída pelos adolescentes do sexo masculino e do feminino, foram identificadas algumas aproximações e diferenças segundo o sexo dos sujeitos. As primeiras delas estão na composição do sistema central,

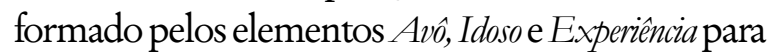
o subgrupo masculino (SM) e por Avô e Idoso para o subgrupo feminino (SF) (Quadro 2 e 3).

Quadro 2 - Distribuição dos elementos segundo frequência de evocação e ordem média de evocação realizadas por adolescentes do sexo masculino. Rio de Janeiro, RJ. 2009.

\begin{tabular}{|c|c|c|c|c|c|c|}
\hline Fm & Elementos & $\mathrm{f}$ & Ome $<2,5$ & Elementos & $\mathrm{f}$ & Ome $>=2,5$ \\
\hline$>=25$ & $\begin{array}{l}\text { AVO } \\
\text { IDOSO } \\
\text { EXPERIÊNCIA }\end{array}$ & $\begin{array}{l}54 \\
47 \\
27\end{array}$ & $\begin{array}{l}2,296 \\
1,702 \\
2,074\end{array}$ & & & \\
\hline$<25$ & $\begin{array}{l}\text { IDADE } \\
\text { CHATA } \\
\text { CONHECIMENTO } \\
\text { COROA }\end{array}$ & $\begin{array}{r}12 \\
6 \\
6 \\
4\end{array}$ & $\begin{array}{l}1,917 \\
2,000 \\
2,333 \\
1,750\end{array}$ & $\begin{array}{l}\text { SABEDORIA } \\
\text { RESPEITO } \\
\text { APOSENTADO } \\
\text { PERSONAGENS } \\
\text { DOENCA } \\
\text { ANTIGO } \\
\text { BENGALA } \\
\text { RUGAS } \\
\text { ACABADO } \\
\text { CAB-BRANCO } \\
\text { REMEDIO } \\
\text { DIFICULDADES } \\
\text { PAI-MAE-TIA } \\
\text { TEMPO }\end{array}$ & $\begin{array}{r}15 \\
12 \\
9 \\
9 \\
7 \\
6 \\
6 \\
6 \\
5 \\
5 \\
5 \\
4 \\
4 \\
4\end{array}$ & $\begin{array}{l}2,800 \\
2,917 \\
3,222 \\
2,778 \\
3,286 \\
2,667 \\
2,500 \\
3,000 \\
2,800 \\
3,200 \\
2,600 \\
3,000 \\
3,000 \\
3,250\end{array}$ \\
\hline
\end{tabular}

Os elementos Sabedoria, Respeito, Personagens, Doença, Bengala, Cabelo Branco e Remédio estão presentes no sistema periférico dos dois grupos. O sistema periférico do SF é acrescido dos elementos Experiencia, Morte, Velhice, Óculos e Conhecimento, enquanto que a periferia do SM é também formada por Aposentado, Antigo, Rugas, Acabado, PaiMãe-Tia, Dificuldades e Tempo.
Essas diferenças entre elementos especificam conteúdos presentes em subconjuntos de significados, em sua maioria compartilhados entre os subgrupos. Os dois subgrupos constituem suas representações com sentidos acerca de ganhos com a velhice (Experiencia, Sabedoria, Conbecimento por SF e Sabedoria por SM), sobre descrição física (Cabelo Branco, Bengala, Óculos porSF e Bengala, Cabelo Branco, Rugas, Acabado por SM), 
condições patológicas (Doença, Morte, Remédio por SFe Doença, Remédio e Dificuldades por SM), sobre uma forma de tratamento (Respeito por SF e SM) e com referência a exemplos de pessoa velha (Personagens por SF e Personagense Pai-Mãe-Tia por SM). Mas também com a referência à Velbice, feita por SF e com o subconjunto Aposentado, Antigo, Tempo peloSM.
A presença do elemento Acabado no sistema periférico do SM parece indicar degeneração, pois que além de uma descrição física, eles qualificam a aparência com o uso de adjetivos como feia, acabada, ruim (corpo), dando um sentido de degradação ao corpo envelhecido.

Quadro 3 - Distribuição dos elementos segundo frequência de evocação e ordem média de evocação realizadas por adolescentes do sexo feminino. Rio de Janeiro, RJ. 2009.

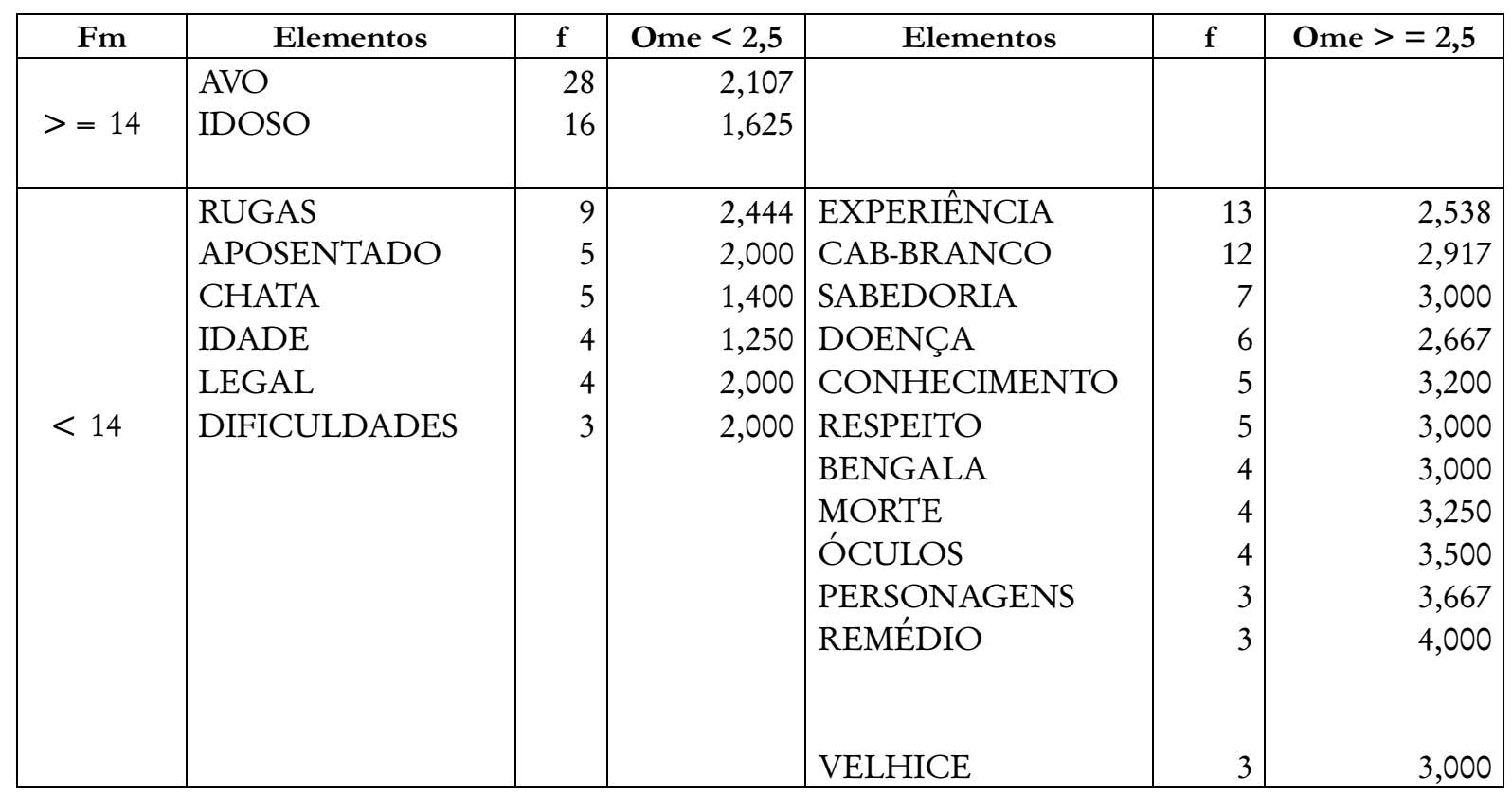

A comparação entre as frequências de indicação de importância e as frequências de evocação de Avô e Idoso para o SF resultou em $64,28 \%$ de marcações de $A v \hat{o}$ evocado, enquanto $31,25 \%$ de Idoso evocado. Desse modo, somente Avô agrega mais um indício de centralidade para SF. Já para o SM, essa comparação sugere que $A v \hat{\text { e }}$ Experiência têm outro indício de centralidade, com $55,5 \%$ e $81,48 \%$ de indicações de importância, enquanto que $I$ doso tem menos do que $50 \%, 44,68 \%$.

O próximo passo foi identificar a organização dos conteúdos da representação com o auxílio da análise de similitude, que permite explorar o número de co-ocorrências entre os elementos evocados através de um índice de contingência. ${ }^{29}$ A leitura cognitiva da árvore de similitude ${ }^{23}$ construída com o corpus total do estudo (figura 1) permite observar que Avô, Idoso e Experiência são elementos que centralizam a formação de três diferentes estrelas - ou seja, de estruturas radiadas com ligação mínima com outros cinco elementos. $^{30}$

A formação estrelar de Avô é composta pelos elementos Dificuldades, Doensa, Remédios, Rugas, Óculos, Chata, Senhora, Gratuidades, Pai-Mãe-Tia e Personagens. Vale ressaltar que estes dois últimos elementos estabelecem com $A v o$ maior valor de conexidade.

Do outro lado do grafo está Idoso, que se associa a Tempo, Experiência, Antigo, Respeito, Senhora, Legal, Aposentado, Acabado e Bengala. Interessante notar que junto a Senhora - único elemento em comum com 
Avô -, os aspectos físicos, como a condição de Acabado e o uso de Bengala, são o subconjunto de significado compartilhado por Avô e Idoso, e que ao mesmo tempo estabelecem uma formação circular integrando esses elementos-centros da organização - Avô-Óculos-Cabelo Branco-Acabado - Idoso-Senhora-Avô. Além disso, observam-se duas formações triangulares, importantes por um detalhamento maior da representação. ${ }^{23}$ Seus vértices ligam os elementos Idoso e Experiência.

Idoso forma um triângulo com Tempo e Experiência e outro com Experiência e Antigo, ao mesmo tempo em que todos eles constituem um círculo - Idoso-Tempo-Experiência-Antigo-Idoso. A conjunção desses triângulos e do círculo sugere que o idoso seja alguém considerado antigo com muita experiência de vida, adquirida com o decorrer do tempo ou por ter maior quantidade de tempo vivido.

A outra estrela observada é a que tem por centro Experiência, que se conecta a cinco cinco elementos - Idade, Conhecimento, Antigo, Idoso e Tempo. Pode indicar que a experiência é um requisito para a obtenção de conhecimento e característica de uma pessoa que é antiga, por ter mais anos de idade e que, portanto, já viveu mais tempo - uma pessoa identificada como idoso.

Os elementos do sistema central são os que estabilizam, unificam e geram os sentidos da representação, por isso estabelecem conexões com os elementos do sistema periférico. ${ }^{15} \mathrm{~A}$ análise de similitude confirma a característica de centralidade de Avô, Idoso e Experiência, que concentram valor simbólico e ao redor dos quais são organizados os componentes da representação. No entanto, são $A v \hat{\text { e }}$ Idoso que dispõem de maior poder de associação, com dez e nove conexões, respectivamente, e sugerem a existência de dois focos de sentidos atribuídos à pessoa velha.

O primeiro deles é centralizado por $A v \hat{o}$. Os elementos relacionados a $A v \hat{o}$ oferecem concretude à imagem de pessoa velha. Isso ocorre com a referência direta a pessoas próximas (avós, outros familiares e conhecidos) ou distantes (artistas e esportistas). Todos parecem ser exemplos e modelos imediatos de pessoa velha compartilhados pela memória coletiva do grupo social de inserção. A construção dessa imagem é detalhada com a menção a aspectos que contemplam uma descrição física, um modo de ser (Chata) e de condições de vivência da velhice. Entre elas estão as patologias e o enfrentamento de dificuldades, que conjuntamente com Gratuidades podem ser observadas no cotidiano dos adolescentes. Seja gratuidade oferecida pela lei no uso dos ônibus, como dificuldades na realização de atividades diárias, as quais podem ser alteradas pela existência de doenças crônicas ou agudas. Como consequência, exigem algum tipo de tratamento, como o uso de medicação. 


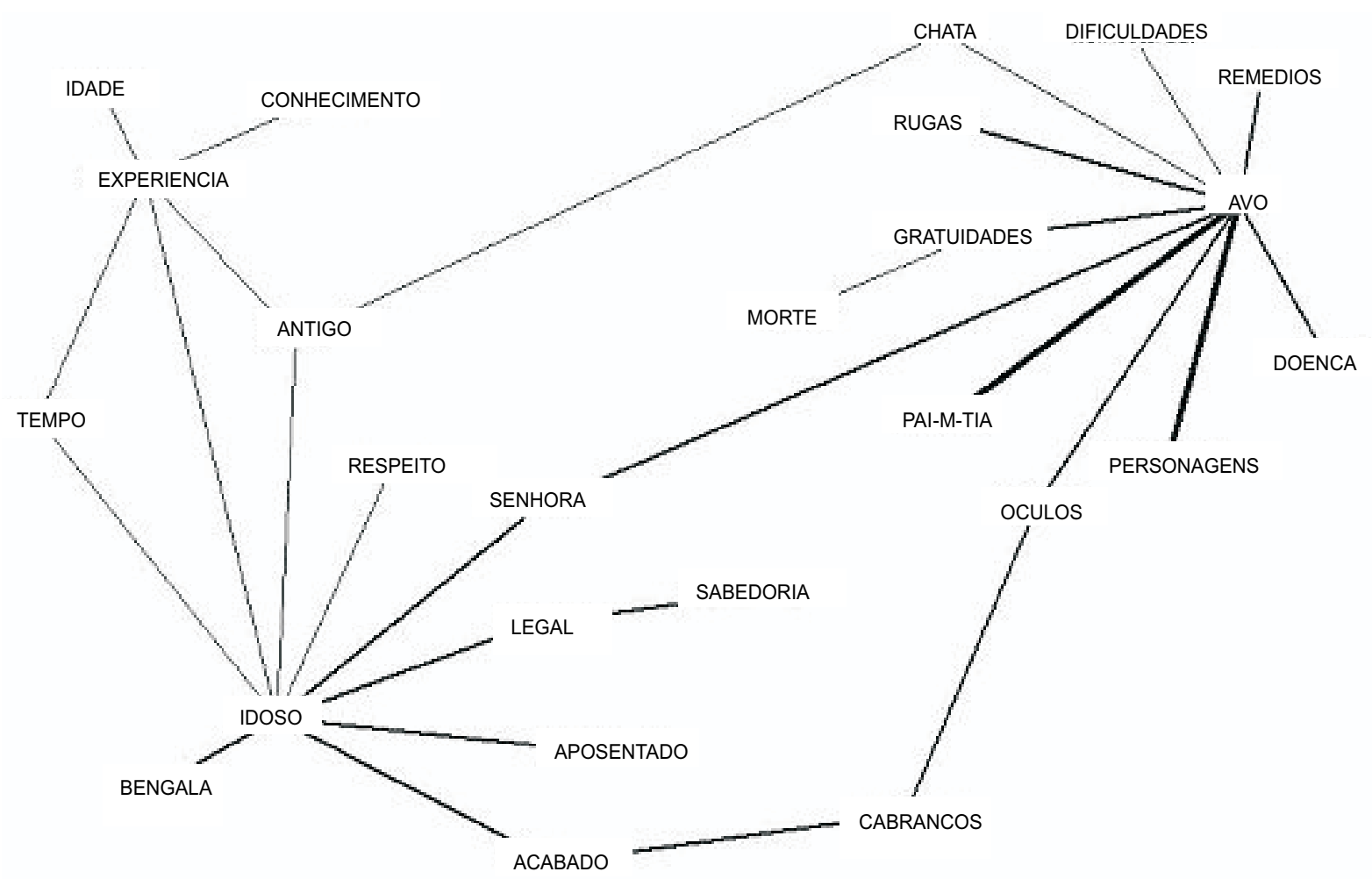

Figura 1 - Árvore máxima de similitude - Total da Amostra. Rio de Janeiro, RJ. 2009.

Idoso, por sua vez, é aquele que vive há mais tempo, por isso é antigo e adquiriu experiência e conhecimento e no atual período da vida está aposentado, após um longo período de trabalho. Ao contrário do $A v \hat{o}$, é uma pessoa legal, que tem sabedoria e merece respeito. Mas, assim como $A v \hat{o}$, tem sua aparência descrita. Pode precisar de uma bengala para se locomover, seu corpo apresenta sinais de decrepitude e de perda da beleza.

Em seguida, foram comparadas a organização da representação dos subgrupos feminino e masculino. Para o SF, Avô confirma sua centralidade ao manter conexão em forma estrelar com 12 elementos na árvore máxima de similitude e ainda compõe cinco triângulos. Avô organiza diretamente a maioria dos subconjuntos de significados presentes na periferia $d a$ representação, com exceção de Experiencia, Sabedoria, Conbecimento (ganhos) e Respeito (forma de tratamento). O que mais se diferencia da árvore de todo o grupo pesquisado e do SM é a ligação direta que estabelece com Idoso, junto ao qual se conecta a Legale Aposentado (figura 2).

Idoso, por sua vez, perde seu poder simbólico de centralidade por se associar apenas a três elementos. Mesmo assim, ele forma um círculo e mantém detalhamento com duas triangulações que conferem precisão entre os elementos Legal, Avô e Aposentado.

Um elemento que se destaca é um componente do sistema periférico próximo, Dificuldades, pois aparece como centro de uma estrela -conecta-se a Avô, Rugas, Bengala, Doença e Respeito. Além disso, é vértice comum na formação de dois triângulos Dificuldades-Avô-Rugas e Dificuldades-Doença-Avô. Este último reforça o sentido de condições patológicas na velhice, que podem ser consideradas por essas adolescentes como causas de dificuldades para uma pessoa velha, exemplificada pelos avós. Essas 
dificuldades podem ser relativas a limitações na realização das tarefas diárias, como de higiene pessoal, preparo da alimentação, deslocamento ou participação em atividades como trabalho e lazer.
As adolescentes podem se referir às doenças como geradoras de dificuldades que limitam a autonomia, as escolhas, impõem nova rotina ou ocasionam dependência.

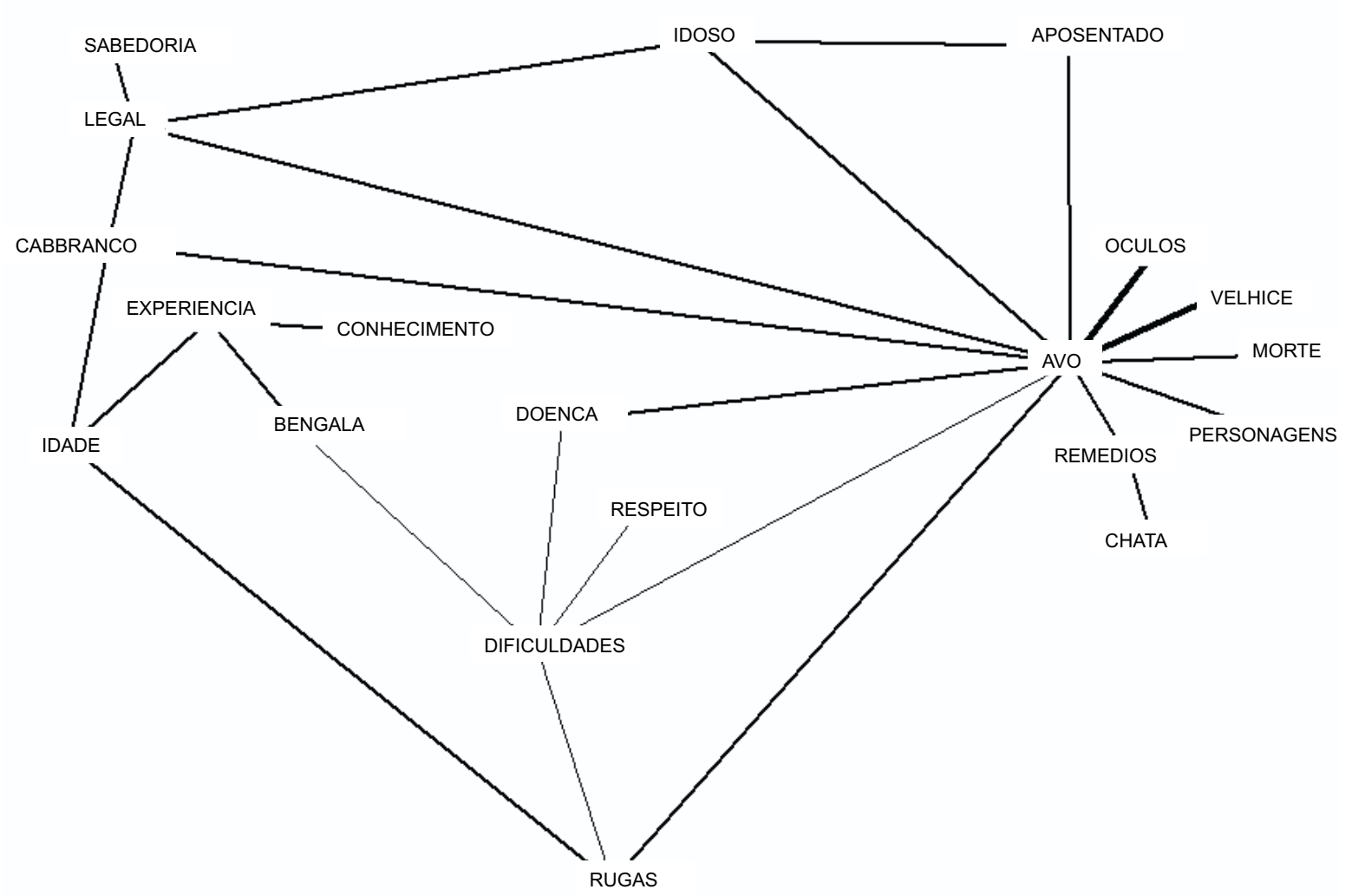

Figura 2 - Árvore máxima de similitude- Subgrupo Feminino. Rio de Janeiro, RJ. 2009.

A árvore de similitude construída com os elementos da representação do SM indica a centralidade de Avôe Idoso como os únicos centros de estrelas, com conexidade com oito e dez elementos, respectivamente (figura 3). Essas conexões se assemelham às da organização $d a$ representação de todo o grupo pesquisado. Mesmo assim, ressalta-se a formação triangular entre Idoso-Aposentado-Coroa e a ligação de Avô com Idoso por meio do elemento Dificuldades. A representação construída pelo SM relaciona a existência de dificuldades tanto à Avô quanto à $I$ doso, associação não realizada pelo SF.

Um dos aspectos que chama a atenção é que, apesar de ter importância indicada pelos sujeitos e de ter frequência e rapidez de evocação para compor o sistema central, Experiência não aparece com poder simbólico central na organização, somente se associa a Idoso. 


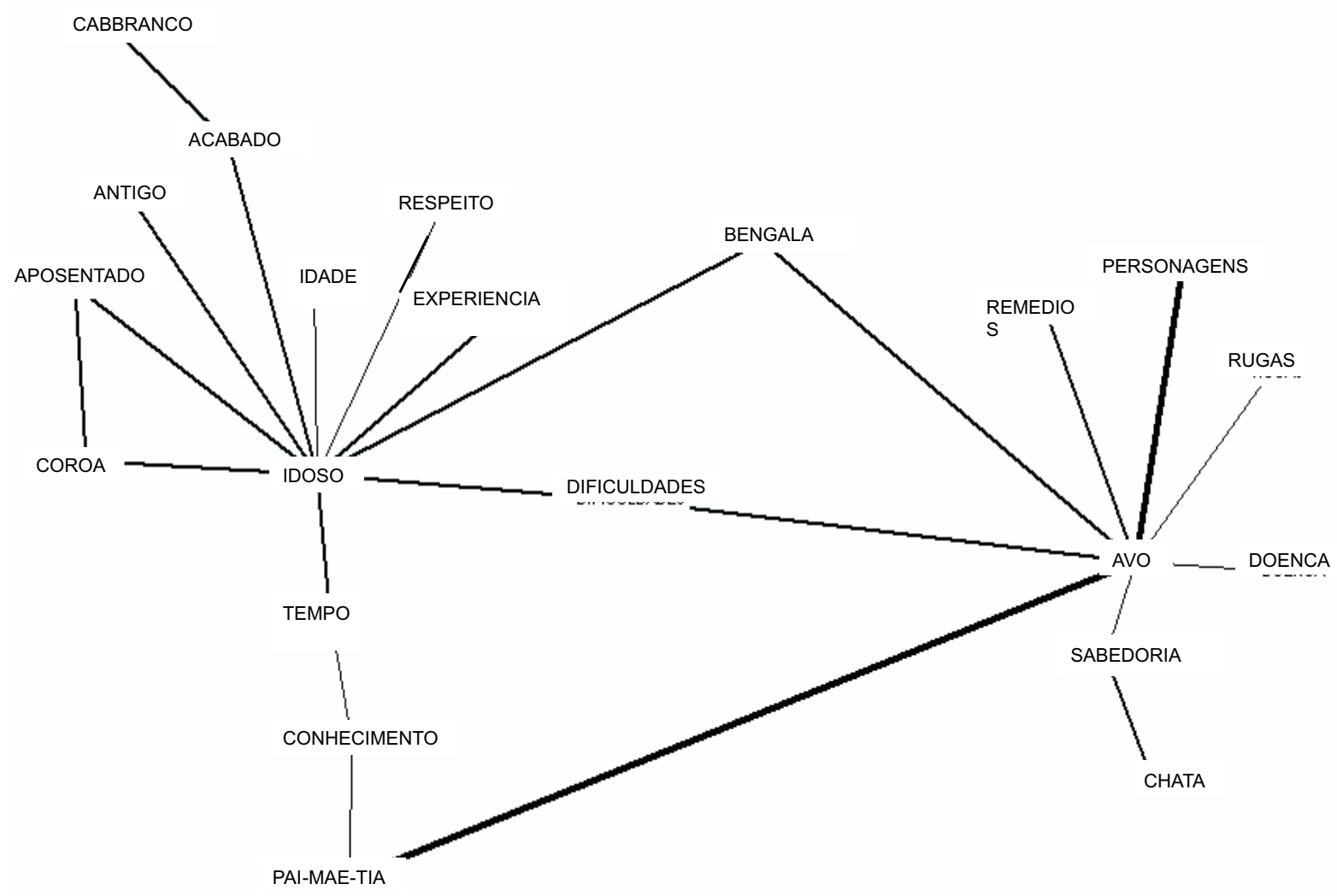

Figura 3 - Árvore máxima de similitude- Subgrupo Masculino. Rio de Janeiro, RJ. 2009.

\section{CONSIDERAÇÕES FINAIS}

O estudo focou-se na compreensão da estrutura e organização dos elementos que compõem os significados da representação social sobre pessoa velha construída por um grupo de adolescentes estudantes e permitiu a comparação da representação segundo a composição do grupo pelo sexo dos participantes.

Os elementos centrais, aqueles que geram e estabilizam a representação, encontrados por meio do TEP são Avô, Idoso e Experiência, dos quais Avô e Experiência tiveram relevância quanto a sua importância e $A v \hat{o}$ e Idoso foram os principais organizadores da representação. Esta, portanto, é centralmente estruturada por uma dimensão normativa, pois seus elementos se acercam de dimensões sócio-afetivas, sociais ou ideológicas, em vez daquela direcionada à realização de tarefas.

Verificou-se que há dois polos de sentidos acerca da pessoa velha: Avô, que parece ser a referência de pessoa velha para esses jovens, seja pela provável convivência com seus avós, por serem considerados importantes e fazerem parte de suas vidas, por serem as pessoas mais velhas que conhecem ou por serem os exemplos de pessoa velha, que circulam nas mídias e em obras literárias, por exemplo. De acordo com essas inferências, a afetividade pode ser uma dimensão que integra essa construção representacional, assim como os aspectos concretos que materializam de forma objetiva uma imagem da velhice. Isso é reforçado tanto pela menção a outros familiares e conhecidos, e a pessoas públicas ou personagens, como pela descrição física. 
O outro polo está centralizado em Idoso, que agrega sentidos relativos a uma construção normativa da categoria Idoso, que enseja respeito e valorização, em oposição a uma conotação negativa ou pejorativa associada ao termo "velho". ${ }^{31}$ Ao Idoso são associados, direta ou indiretamente, elementos considerados em outros estudos como ganhos com o envelhecer experiência, sabedoria, conhecimento -, que compensariam perdas e limitações. ${ }^{9-13}$ Estas são os aspectos em comum entre Idoso e $A v \hat{o}$, ou seja, o declínio e limitações físicas, elementos frequentemente encontrados em estudos similares. ${ }^{9-14}$

O corte de acordo com os sexos dos participantes identificou duas diferentes representações segundo o SF e o SM. A construída por SF tem sua estabilidade e organização centralizada por $A v \hat{\text {, e }}$ a elaborada por SM, por Avô e Idoso. Ao mesmo tempo, há semelhanças entre os sentidos formadores dos

\section{REFERÊNCIAS}

1. Jodelet D. Representações sociais: um domínio em expansão. In: Jodelet D. As representações sociais. Rio de Janeiro: EDUERJ; 2001. p. 17-44.

2. Moscovici S. Representações sociais: investigações em psicologia social. 5. ed. Petrópolis: Vozes; 2007.p.404

3. Kalache A, Veras RP, Ramos LR. O envelhecimento da população mundial: um desafio novo. Rev Saud Pública 1987 jun; 21(3): 200-10.

4. Veras RP, Ramos LR, Kalache A. Crescimento da população idosa no Brasil: transformações e conseqüências na sociedade. Rev. Saúde Pública 1987 jun; 21 (3): 225-33.

5. Veras RP. A longevidade da população: desafios e conquistas. Serviço Social e Sociedade 2003 set; 24(75): 5-18.

6. Veras RP. A novidade da agenda social contemporânea: a inclusão do cidadão de mais idade. A Terceira Idade 2003 set; 14(28): 6-29.

7. Veras RP, Caldas CP. Promovendo a saúde e a cidadania do idoso: o movimento das universidades da terceira idade. Ciênc Saud Colet 2004 abr/jun; 9(2): 423-432. conteúdos mais flexíveis às características do contexto imediato, ou seja, os que compõem o sistema periférico.

No entanto, a organização dos elementos da representação construída pelo subgrupo feminino sugere maior especificidade da relação do elemento central Avô com os outros elementos, assim como detalhamento com a presença de diferentes triângulos. Destaca-se que, apesar de os participantes dos dois subgrupos serem jovens e estarem temporalmente longe da velhice, essas relações não são realizadas pelo subgrupo masculino, ressaltando assim maior complexidade na construção de sentidos atribuídos à pessoa velha pelo subgrupo feminino.

Como perspectiva futura de análise, as concepções de gênero podem ser parâmetros úteis para possíveis explicações entre essas diferenças e semelhanças encontradas nas representações elaboradas por esses dois subgrupos.

8. Hummel C. Représentations de la vieillesse chez des jeunes adultes et des octogénaires. Gérontologie et société 2001 set; 3(98): 239-252.

9. Martins CRM, Camargo BV, Biasus F. Representações sociais do idoso e da velhice de diferentes faixas etárias. Universitas Psychologica 2009 set/dez; 8 (3): 831-847.

10. Mithidieri O, Tura LFR. Os sentidos de idoso para estudantes de medicina: subsídios para a seleção de conteúdos curriculares. In: Textos completos da 3. Jornada Internacional e 1. Conferência Brasileira sobre representações sociais; 2003 set 2 5, Rio de Janeiro. Rio de Janeiro: Universidade do Estado do Rio de Janeiro; 2003. p. 605-619.

11. Sousa L, Cerqueira M. As imagens da velhice em diferentes grupos etários: um estudo exploratório na população portuguesa. Revista Kairós 2005 dez; 8(2): 189-206.

12. Teixeira MCTV, et al. Envelhecimento e rejuvenescimento: um estudo de representação social. Rev Bras Geriatr Gerontol 2007; 10(1): 49-71.

13. Wachelke JF, et al. Princípios organizadores da representação social do envelhecimento: dados coletados via internet. Estudos de Psicologia 2008 mai/ago; 13(2): 107-116. 
14. Arnold-Cathalifaud M, et al. La vejez desde la mirada de los jóvenes chilenos: estudio exploratorio. Ultima Década $2007 \mathrm{dez}$; 27: 75-91.

15. Sá CP. Núcleo central das representações sociais. 1. ed. Petrópolis: Vozes; 1996. p.189

16. Abric JC. L'analyse structurale des representations sociales. In: Moscovici S, Buschini F. Lês méthods des sciences humaines. Paris: PUF; 2003. p. 375-92.

17. Cromack LMF, Bursztyn I, Tura LFR. O olhar do adolescente sobre a saúde: um estudo de representações sociais. Ciênc Saud Colet 2009 mar/abr; 14(2): 627-634.

18. Goetz E, et al. Representação social do corpo na mídia impressa. Psicol. Soc 2008 mai/ago; 20(2): 226-236.

19. Moreira MASP, et al. Pensando a saúde na perspectiva dos imigrantes brasileiros em Portugal. Revista Gaúcha de Enfermagem 2007 dez; 28(4): 527-533.

20. Oltramari LC, Camargo BV. Representações sociais de mulheres profissionais do sexo sobre a AIDS. Estudos de Psicologia 2004 maio/ago; 9(2): 317-323.

21. Tura LFR, et al. Representações sociais de hepatite e profissionais de saúde: contribuições para um (re)pensar da formação. Ciência, Cuidado e Saúde 2008 abr/jun; 7: 207-215.

22. Vergès $\mathrm{P}$. Os questionários para análise das representações sociais. In: Moreira ASP, et al. Perspectivas teórico-metodológicas em representações sociais. João Pessoa: Universidade Federal da Paraíba; 2005. p. 201-228.

23. Pereira FJC. Análise de dados qualitativos aplicados às representações sociais. In: Moreira
ASP, et al. Perspectivas teórico-metodológicas em representações sociais. João Pessoa: Universitária ; 2005. p. 25-60.

24. Bardin L. L'analyse de contenu et de la forme des comunications. In: Moscovici S, Buschini F. Lês méthods des sciences humaines. Paris: PUF; 2003. p. 243-270.

25. Conselho Nacional de Saúde(Brasil). Resolução n. ${ }^{\circ} 196$, de 10 de outubro de 1996: "Aprova diretrizes e normas regulamentadoras de pesquisas envolvendo seres humanos". Cad Ética em Pesquisa 1998;1(1):34-46.

26. Flament C. Estrutura e dinâmica das representações sociais. In: Jodelet D. As representações sociais. Rio de Janeiro: EDUERJ; 2001.p. 173-186.

27. Ginzburg C. Mitos, emblemas, sinais: morfologia e história. 3. ed. São Paulo: Companhia das Letras; 1989. p.288

28. Campos PHF. Educação social de rua: estudo estrutural de uma prática político-social. $\mathrm{O}$ Social em questão 2003 jun; 9(9): 28-48.

29. Bouriche B. L' analyse de similitude. In: Abric JC. Méthodes d'étude des représentations sociales. Saint-Agne: Éditions Érès; 2003. p. 221252.

30. Tura LFR. AIDS e prevenção: a estrutura das representações sociais. In: Madeira MC, Jodelet D. AIDS e representações sociais: à busca de sentidos. Natal: EDUFRN;1998. p. 121-154.

31. Peixoto C. Entre o estigma e a compaixão e os termos classificatórios: velho, velhote, idoso, terceira idade. In: Barros MML. Velhice ou terceira idade: estudos antropológicos sobre identidade, memória e política. Rio de Janeiro: FGV; 2000. p. 69-84. 